\title{
Effects of early mobilization (EM) in patients with noninvasive positive pressure ventilation (NIPPV) in Intensive Care Unit (ICU): a randomized controlled trial
}

\section{Yanhao Wang}

Key Laboratory of Shaanxi Province for Craniofacial Precision Medicine Research, College of Stomatology, Xi'an Jiaotong University, 98 XiWu Road, Xi'an, 710004, Shaanxi, PR China

\section{Zixuan Huang}

College of Nursing, Chongqing Medical University, Chongqing, China.

\section{Jun Duan}

Department of Respiratory Medicine, The First Affiliated Hospital of Chongqing Medical University, Chongqing, China

\section{Wenxin Xu}

College of Nursing, Chongqing Medical University, Chongqing, China.

\section{Jiayi Mao}

College of Nursing, Chongqing Medical University, Chongqing, China.

\section{Xiaoying Yan}

College of Nursing, Chongqing Medical University, Chongqing, China.

\section{Qin Yang}

College of Nursing, Chongqing Medical University, Chongqing, China.

Jianli Chen

College of Nursing, Chongqing Medical University, Chongqing, China.

Shaoyu Mou ( $D$ shaoyumou@163.com )

College of Nursing, Chongqing Medical University, Chongqing, China.

\section{Rong Lan}

College of Stomatology, Chongqing Medical University, Chongqing 401147

\section{Research Article}

Keywords: Early Mobilization (EM), Noninvasive positive pressure ventilation (NIPPV), Intensive Care Unit (ICU), Randomized controlled trial (RCT)

Posted Date: February 16th, 2021 
DOI: https://doi.org/10.21203/rs.3.rs-132343/v1

License: (c) (1) This work is licensed under a Creative Commons Attribution 4.0 International License. Read Full License 


\section{Abstract \\ Background}

Early mobilization (EM) may be an effective intervention for the promotion of rehabilitation in noninvasive positive pressure ventilation (NIPPV) patients. The aim of this study was to investigate the effects of EM in patients with NIPPV in Intensive Care Unit (ICU).

\section{Methods}

Participants were randomly allocated to the intervention group involving active and passive activities combined with routine treatments and the control group with routine treatments in this single-center, parallel-designed randomized controlled trial. Participants accepted initiative and passive activities following brought by medical and nursing team who were standardized training The primary outcomes were the incidence of ICU-AW, length of ICU stay, duration of ventilation and mortality.

\section{Results}

There were no adverse event in participants during EM. Compared with the control group, there was a significantly lower length of ICU stay ( $m=6.0$ vs 7.8 days, respectively, $P=0.038$ ), incidence of ICUacquired weakness ( $n=17.4 \%$ vs $50 \%$, respectively, $P=0.026)$, duration of ventilation ( $m=2.1$ vs 4.0 days, respectively, $P=0.019)$ in the intervention group.

\section{Conclusions}

EM is feasible and safe in noninvasive ventilator patients, it can decrease the incidence of ICU-acquired weakness, length of stay and duration of noninvasive ventilation in ICU, and promoted the recovery of grip strength.

\section{Introduction}

Long-term bed rest or prolonged immobility can lead to serious complications in critically ill patients undergoing mechanical ventilation, such as ICU-acquired weakness (ICU-AW), cardiac circulatory system dysfunction, metobolic and mental disorders etc ${ }^{3-4}$. These complications not only prolong the duration of mechanical ventilation and ICU hospitalization, but also seriously impair the rehabilitation of patients' body functions ${ }^{5-7}$. As one of the most common complications, ICU-AW is clinically manifested by limb paralysis, muscle atrophy and difficulty in ventilator weaning ${ }^{8}$. It has been reported that the incidence of ICU-AW among patients with mechanical ventilation could be $33 \%-82 \%{ }^{9-10}$. ICU-AW also prolongs the time of ICU hospitalization, what's worse, it decreases the patient's life quality during 90 days after 
discharge ${ }^{11}$. Previous studies had followed up a queue of survivors of ARDS, finding that even 5 years after discharge, physical dysfunction failed to recover completely ${ }^{5}$. At present, no pharmacological therapy are recommended to prevent and treat ICU-AW ${ }^{12}$. The managements mainly focus on supportive care for patients. ${ }^{13}$

Recently, it is suggested that long-term bed-rest, the traditional nursing mode, should be changed among mechanical ventilation patients. Meanwhile, early mobilization (EM) is recommended in many guidelines as a preferable method to promote the recovery. Even though EM is thought to be feasible and beneficial for mechanical ventilation patients in the ICU ${ }^{14,15}$, it is poorly defined and previously only referred to early program of standing and walking training (rehabilitation) or simply moving patients' limbs, etc. ${ }^{16}$.

Many studies have shown the effectiveness of EM, as it not only helps patients regain muscle strength, especially inspiratory muscle, which leads to better physical function ${ }^{17,18}$, but also prolongs patients' ventilator-free time and increases their ability to ambulate and to conduct daily activities ${ }^{19}$. However, EM may also give rise to occasional side effects, such as increases of heart rate and respiratory rate and to minor adverse events, such as transient hypotension, oxygen desaturation, patient-ventilator asynchrony and agitation. Few studies reported that EM in AECOPD patients during hospitalization may increase the mortality and there are some risks and potential dangers such as unscheduled extubation ${ }^{24}$. The underlying cause may be that the critical conditions make these patients more vulnerable to accept $\mathrm{EM}^{2}{ }^{24}$.

Non-invasive ventilation provides an "intermediate" or "transitional" alternative of auxiliary ventilation between oxygen therapy and invasive ventilation, especially for respiratory failure in early stage or without emergent tracheal intubation indications ${ }^{26}$. Therefore, comparing with invasive ventilation patients, non-invasive ventilation patients may have better conditions and tolerance for EM, the safety of EM in non-invasive ventilation patients might be better. Moreover, interventions such as early and sustained mobilization may be key in deterring the progression to intubation and invasive mechanical ventilation in NIPPV patients. Hodgson ${ }^{26}$ even emphasized the safety of EM among patients with mechanical ventilation, and gave some suggestions on the safety standards: it is necessary to organize a multidisciplinary treatment team to ensure the safety and effectiveness of EM.

Many studies have demonstrated the feasibility and safety of EM for invasive mechanical ventilation ICU patients ${ }^{21}$ since EM can prevent ICU-AW by increasing the duration and level of active exercises and reducing the duration of ventilation and length of ICU stay ${ }^{28}$. However, few studies have reported whether EM has the similar effect for non-invasive ventilation population, especially NIPPV patients. To figure out this, we formed a multidisciplinary team including doctors, nurses and respiratory therapists in this study. Meanwhile, we assessed the patients' conditions before EM to ensure the safety. This study aimed to investigate the feasibility and safety of EM in NIPPV patients and the effect of EM on the promotion of rehabilitation in patients with NIPPV in ICU. Therefore, we conducted a randomized controlled trail to 
investigate whether EM can prevent the ICU-AW, reduce the duration of (non-invasive) ventilation and the length of ICU stay, and improve the physical function among NIPPV patients.

\section{Results}

From July, 2017 to March, 2019, our team screened 481 patients, 48 of them were finally enrolled and assigned randomly at 1:1 ratio to the intervention group and the control group.

During the study, 1 patient in the intervention group and 3 patients in the control group refused to join in further research and were considered to be "drop out". Moreover, another 3 patients in the control group were excluded after enrollment because two of them were respectively diagnosed with influenza and tuberculosis; 1 patient was severely restricted to movements with aggravating heart failure. Eventually, 41 patients completed the whole procedure (23 participants in intervention group, 18 participants in control group). The trial profile is presented in Fig. 1 (Fig. 1).

Table 2 shows the baseline parameters and primary diseases of the study population (Table 2). The average of APACHE II score, creatinue and leukocyte for the intervention group were $18,58 \mathrm{mmol} / \mathrm{L}$, $7.9 \times 10^{9} / \mathrm{L}$ respectively, while those of the control group were $16.3,56.5 \mathrm{mmol} / \mathrm{L}, 9.3 \times 10^{9} / \mathrm{L}$ respectively. There was no significant difference in demographic data and primary diagnosis between the intervention and control groups $(p>0.05)$.

Compared with the control group, durations of ICU stay and ventilation were significantly shorter $(\mathrm{p}=$ $0.038, p=0.019$ respectively), and the incidence of ICU-acquired weakness was lower in the intervention group. Moreover, the increase of right or left hand-grip strength was significantly higher in intervention group than that of control group ( $p=0.00, p=0.047$ respectively) (Table 3 ). No adverse events were found during the study and 1 patient in the control group passed away because of respiratory failure caused by severe pneumonia. Statistically, there was no significant difference in ICU mortality $(p=0.439)$.

\section{Discussion}

In this study, alle th participants in the intervention group carried out EM personalized according to their physical tolerance. During the procedure, no adverse events such as falls or unplanned extubation occurred, and there was no significant difference in mortality between the two groups $(p>0.05)$. The results indicated that EM for patients with non-invasive positive pressure ventilation in ICU was safe under the conditions of this study.

EM may be an effective strategy to prevent ICU-acquired weakness, as it not only enhances muscle strength and prevents muscle atrophy, but also reduces oxidative stress and inflammation ${ }^{38}$. EM has a positive influence on the recovery of muscle function and the reduction of the occurrence of ICU-AW. In this study, patients with non-invasive positive pressure ventilation were provided with EM including passive activities such as joint flexion, abduction, rotation and turning over in bed, as well as initiative activities, such as grip strength training, upper limb strength training and bedside standing. We used MRC 
score to evaluate the occurrence of ICU-acquired weakness. The results of this study showed that the incidence of ICU-Acquired weakness and the duration of ICU stay in the intervention group were significantly lower than those in the control group $(P=0.026, P=0.0038$ respectively), the increase of left and right hand grip strength in the intervention group were significantly higher than that in the control group $(P=0.047, P=0.00$ respectively). It was confirmed that EM can reduce the occurrence of ICUacquired weakness, promote the recovery of muscle strength and shorten the length of ICU-stay in patients receiving NIPPV.

Ventilation is an effective treatment for respiratory failure, but it can also lead to diaphragmatic atrophy and contractile dysfunction, namely ventilation-related diaphragmatic dysfunction (VIDD). Animal experiments have shown that mechanical ventilation can cause diaphragm contraction dysfunction in mice in only 6 hours ${ }^{39}$. Some studies have shown that prolonged mechanical ventilation can lead to difficulty in weaning and poor prognosis that will further restrict the activity of patients, thus further causing muscle (including diaphragm) atrophy, vicious circulation and aggravating ICU-Acquired weakness ${ }^{40-42}$. The results of this study showed that the duration of noninvasive ventilation in the EM intervention group was significantly lower than that in the control group $(p=0.019)$. The reason behind may be that EM prevented the occurrence of VIDD in patients with noninvasive ventilation. However, due to the lack of direct evaluation of diaphragm contraction function in this study, whether EM could prevent diaphragm atrophy and the occurrence of VIDD still needs further study.

This study promoted the recovery of physical function in patients with NIPPV in ICU through EM. But at present, there are many factors restricting the implement of EM such as the lack of breathing therapists and physiotherapists, inadequate muscle strength training equipment, lack of awareness of patients and medical staff towards EM and so on. In some extent, these factors limit the development of EM, which should be solved in the future. The results of this study showed that the EM can significantly improve the patient's body function and beneficial for them, we suggest that EM can be added to the routine management program for patients, especially for patients with better activities tolerance. Patients receiving NIPPV represent an understudied group. Studies have shown that the use of NIPPV can improve the physical activity in COPD patients ${ }^{43-45}$, therefore, early mobilization and NIPPV may have a synergistic positive effect on patients. Moreover, interventions such as early and sustained mobilization may be key in deterring the progression to intubation and mechanical ventilation, EM may also be an effective measure to improve the long-term prognosis of patients. Further investigation in this area can start from better intervention planning and longer follow-up to provide more valid evidence.

\section{Methods}

\section{Participants}

The trial had been registered on the World Health Organization International Clinical Trial Registry and the Chinese Clinical Trial Registry (No. ChiCTR1900021797) in 2019/03/10. We recruited study participants in The First Affiliated Hospital of Chongqing Medical University (Chongqing, China). The institutional 
review board of the hospital approved the study and written informed consent was obtained from the participant or his her authorized representative.

The inclusion criteria of EM: (1) Patient ought to be aged 18 years or older; (2) Patient who has hospitalized in ICU received NIPPV for less than 48h; (3) The projected time of NIPPV was not less than 24h. (4) Patient ought to be conscious and cooperative.

The exclusive criteria of EM: (1) GCS score was not less than or equal to 12; (2) Patient who was unable to move or be moved; (3) Patient who has received non-invasive positive pressure ventilation for more than $48 \mathrm{~h}^{28}$; (4) Patient who had an incurable disease was projected six-month mortality rate more than or equal to50\%; (5) Patient who has received invasive mechanical ventilation before NIPPV; (6) Patient who cannot be up to the standard of EM; (7) Patient who was suffering from severe contagious diseases.

Patients in the medical ICU were screened daily to identify adults ( $\geq 18$ years of age) who had been on mechanical ventilation for less than $72 \mathrm{~h}$, were expected to continue for at least $24 \mathrm{~h}$, and who met criteria for baseline functional independence. A participant's daily screening results appear one item in the exclusive criteria of EM, the participant would be excluded. An adverse event emerges, the trial would be stopped.

\section{Sample size}

This study is a pilot study to establish the feasibility and safety and to support our future study about EM in NIPPV patients. According to Hodgson's previous feasibility studies in severely ill ICU patients ${ }^{1}$, a minimum of 20 patients assigned to per group was deemed necessary to avoid the occasional loss of follow-up and facilitate meaningful assessment of feasibility and safety.

\section{Procedures}

Generated by computer, random numbers were randomly assigned in a 1:1 ratio to the intervention group and the control group. We used the random envelope method to put the plan of the intervention group and the control group into a light-proof envelop coded in order. It was given to investigators after sealed. When the eligible patient showed up, investigators coded the patient as the order of random numbers (ascending sequence), and then opened the corresponding envelope, and took measures according to the plan shown in the envelope. It included 24 cases of the intervention group (EM combined with standard treatment and nursing measures of ICU) and 24 cases of the control group (standard treatment and nursing measures of ICU). The total number of participants was 48 . Blinding was conducted in patients and the researchers who performed the measures.

Before each intervention, the researchers would comprehensively assess the patient's condition including the following items ${ }^{29,30}$, (1)blood pressure increases or decreases suddenly with mean arterial blood pressure lower than $65 \mathrm{mmHg}$ or higher than $110 \mathrm{mmHg}$; (2)heart rate $(\mathrm{HR}) \geq 130$ beats $/ \mathrm{min}$ or $\leq 50$ beats/min; (3) respiratory rate (RR) $\leq 8 \mathrm{times} / \mathrm{min}$ or $\geq 40$ times/min (4) pulse oxygen saturation $\left(\mathrm{SPO}_{2}\right)$ $\leq 90 \%$ (5) patients' discomfort which fails them to accept intervention any more (6) clinical 
manifestations or signs of cardiopulmonary distress (7)severe adverse events including falling out of bed, unexpected pulling out of tuber, sudden coma, malignant arrhythmia, ischemia myocardial, acute respiratory distress (ARDS), etc. Once any of these items occurred, the EM of the intervention group would be suspended. While during each implementation, researchers would closely monitor the changes of various indicators for the participants to ensure the safety.

In order to improve the muscle strength of participants to prevent ICU-acquired weakness, participants accepted initiative and passive activities following brought by medical and nursing team who were standardized training. Before those activities, patients were required to test their grip strength by grippers. As the definition of EM still needs further evidence to underpinned ${ }^{16}$, in this study EM comprises of passive and initiative activities, moving patients' limbs and sitting them out of bed $28,31,32$, these were sequential steps and the intervention providers had been trained in consistency. Passive activities ${ }^{28,32-}$ 36: In the begin, the intervention providers flexed and extended the patient's fingers for 30 times each hand, secondly rotated the wrist clockwise, and promoted elbow flexion and extension, thirdly, completed abduction and flexion of each shoulder, and then promoted internal rotation and external rotation of each shoulder. Besides, from the distal end of lower extremity, providers helped patient complete rotation of ankle joint clockwise, flexion and extension of knee joint to promote blood circulation of limbs, and improve muscle strength. In the end, providers would assist patients to turn over in bed. Initiative activities $32-35$ : In the begin, soft elastic balls were applied for patients to enhance their hand grip strength at an average speed of 30 times per minute, and then they were required to lift $1 \mathrm{~kg}$ dumbbell at an average speed of 25 times per minute to exercise upper arm strength. Thirdly, participants completed the lower extremity initiative activity including rotation of ankle joint clockwise, flexion and extension of knee joint 10 times each per minute to improve muscle strength. At last, patients would be asked to sit by the bed without any support for 10-20 minutes according to their tolerance degree of the activity. In the intervention group, participants carried out all the activities twice a day, 30 minutes each time (Table 1). Except for EM, participants in the control group received the same level of clinical care as the intervention group (standard of care).

During the procedure of the trial, each participant in the intervention group was equally distributed with the same duration of those activities even though the intensity of each item of those activities depended on patients' tolerance levels and their physical condition assessment. In order to prevent adverse events in the course of the trial, the relevant knowledge and skills for the researchers would be assessed regularly, and the operation of EM would be regularly examined. If any adverse events occur to the subjects, any activities must be suspended immediately and handled promptly according to the doctor's instructions.

\section{Variables and measures}

As for the primary outcome, it consisted of efficacy indicators and safety indication of EM implementing. Efficacy indicators include: (1) occurrence of ICU acquired weakness (ICU-AW) (2) the length of ICU stay (3) duration of NIPPV; Safety indicators include ICU mortality rate and occurrence of adverse events. The 
secondary outcome was the growth value of hand-grip strength. The calculation method was to subtract the grip strength at discharge from the grip strength at admission.

The occurrence of ICU-Acquired weakness is evaluated by MRC muscle strength score, which means the muscle strength of limbs would be comprehensively evaluated. ICU-acquired weakness was diagnosed when the muscle strength score is lower than 48 credits and also lower than the patient's credits assessed before each intervention patient had in the group ${ }^{37}$, researchers would observe and record the vital signs and indicators to monitor the process of patient's conditions consistently. All the outcomes were measured by medical personnel.

\section{Ethics Statement}

This was a single-center, parallel-designed randomized controlled trial in the respiratory intensive care unit (RICU) of the First Affiliated Hospital of Chongqing Medical University (Chongqing, China). The research plan and informed consent submitted in this project complied with the medical ethics principles and the requirements of the Helsinki Declaration. The research design based with science evidence does not bring unnecessary risks to the participants, and maximum protection had been given to ensure the safety and privacy of the subjects. The trial was approved by the Ethics Committee of the First Affiliated Hospital of Chongqing Medical University (Document No. 2018-015).

\section{Statistical analysis}

We utilized SPSS software (version 19.0) to analyze the data. Continuous variables were described as mean and standard deviation or as median (interquartile range). Kolmogorov-Smirnov test was used for testing the normality of distribution unpaired. Normally distributed variables were compared by independent Student's $t$ tests. All statistical tests were two-sided, and significance was determined at the 0.05 probability level and non-normally variables were compared by Wilcoxon, Mann-Whitney U tests. We used the $\chi^{2}$ test to compare categorical variables between the intervention group and control group.

\section{Abbreviations}

RCT, randomized controlled trial; NIPPV, noninvasive positive pressure ventilation; EM, early mobilization; ICU, intensive care unit; RICU, Respiratory intensive care unit; ICU-AW, ICU-acquired weakness; COPD, chronic obstructive pulmonary diseases; ARDS, acute respiratory distress syndrome; AECOPD, acute exacerbation of chronic obstructive pulmonary disease; GCS, Glasgow coma scale; ROS, reactive oxygen species; VIDD, ventilation-related diaphragmatic dysfunction.

\section{Declarations}

\section{Conflicts of Interest:}

Yanhao Wang, Zixuan Huang, Rong Lan, Jun Duan, Wenxing Xu, Jiayi Mao, Xiaoying Yan, Qin Yang, Jianli Chen, Shaoyu Mou declared no conflict of interest. 
Acknowledgement:

This study was funded by Scientific Research and Innovation Experiment Project of Chongqing Medical University (CXSY201720).

\section{Author contributions:}

In this study, Y.W., Z.H., J.D. and S. M. contributed to the conception and design of the study. Y.W. and Z.H. searched the literatures, Z.H., J.D., W.X., J. M., X.Y., Q.Y. and J.C. performed the investigation and collected the data. Y.W. analysed the data. Y.W., R.L. and Z.H. prepared the manuscript.

\section{Additional Information:}

Yanhao Wang, Zixuan Huang, Rong Lan, Jun Duan, Wenxing Xu, Jiayi Mao, Xiaoying Yan, Qin Yang, Jianli Chen, Shaoyu Mou have read and approved the final manuscript and the authors declared no conflict of interest.

\section{References}

1. Hodgson, C. L. et al. A randomised controlled trial of an open lung strategy with staircase recruitment, titrated PEEP and targeted low airway pressures in patients with acute respiratory distress syndrome. Crit Care. 15 (3), R133 (2011).

2. Shehabi, Y. et al. Early goal-directed sedation versus standard sedation in mechanically ventilated critically ill patients: a pilot study*. Crit Care Med. 41 (8), 1983-1991 (2013).

3. Vincent, J. L. \& Norrenberg, M. Intensive care unit-acquired weakness: framing the topic. Crit Care Med. 37 (10 Suppl), S296-8 (2009).

4. Clavet, H., Hebert, P. C., Fergusson, D., Doucette, S. \& Trudel, G. Joint contracture following prolonged stay in the intensive care unit. CMAJ. 178 (6), 691-697 (2008).

5. Herridge, M. S. et al. Functional disability 5 years after acute respiratory distress syndrome. N Engl J Med. 364 (14), 1293-1304 (2011).

6. De Jonghe, B., Bastuji-Garin, S., Sharshar, T., Outin, H. \& Brochard, L. Does ICU-acquired paresis lengthen weaning from mechanical ventilation? Intensive Care Med. 30 (6), 1117-1121 (2004).

7. Garnacho-Montero, J., Amaya-Villar, R., Garcia-Garmendia, J. L., Madrazo-Osuna, J. \& Ortiz-Leyba, C. Effect of critical illness polyneuropathy on the withdrawal from mechanical ventilation and the length of stay in septic patients. Crit Care Med. 33 (2), 349-354 (2005).

8. Bloch, S., Polkey, M. I., Griffiths, M. \& Kemp, P. Molecular mechanisms of intensive care unit-acquired weakness. Eur Respir J. 39 (4), 1000-1011 (2012).

9. Griffiths, R. D. \& Hall, J. B. Exploring intensive care unit-acquired weakness. Preface. Crit Care Med. 37 (10 Suppl), S295 (2009). 
10. Castro, E., Turcinovic, M., Platz, J. \& Law, I. Early Mobilization: Changing the Mindset. Crit Care Nurse. 35 (4), e1-5 quiz e6 (2015).

11. Rydingsward, J. E. et al. Functional Status in ICU Survivors and Out of Hospital Outcomes: A Cohort Study. Crit Care Med. 44 (5), 869-879 (2016).

12. Shepherd, S. J., Newman, R., Brett, S. J. \& Griffith, D. M. Pharmacological Therapy for the Prevention and Treatment of Weakness After Critical Illness: A Systematic Review. Crit Care Med. 44 (6), 11981205 (2016).

13. Kramer, C. L. Intensive Care Unit-Acquired Weakness. Neurol Clin. 35 (4), 723-736 (2017).

14. Bourdin, G. et al. The feasibility of early physical activity in intensive care unit patients: a prospective observational one-center study. Respir Care. 55 (4), 400-407 (2010).

15. Hodgson, C. et al. Early mobilization and recovery in mechanically ventilated patients in the ICU: a binational, multi-centre, prospective cohort study. Crit Care. 19, 81 (2015).

16. Bernhardt, J., English, C., Johnson, L. \& Cumming, T. B. Early mobilization after stroke: early adoption but limited evidence. Stroke. 46 (4), 1141-1146 (2015).

17. Nava, S. Rehabilitation of patients admitted to a respiratory intensive care unit. Arch Phys Med Rehabil. 79 (7), 849-854 (1998).

18. Needham, D. M. Mobilizing patients in the intensive care unit: improving neuromuscular weakness and physical function. JAMA. 300 (14), 1685-1690 (2008).

19. Files, D. C. et al. Therapeutic exercise attenuates neutrophilic lung injury and skeletal muscle wasting. Sci Transl Med. 7 (278), 27832 (2015).

20. Nava, S. Rehabilitation of patients admitted to a respiratory intensive care unit. Arch Phys Med Rehabil. 79 (7), 849-854 (1998).

21. Hodgson, C. L. et al. A Binational Multicenter Pilot Feasibility Randomized Controlled Trial of Early Goal-Directed Mobilization in the ICU. Crit Care Med. 44 (6), 1145-1152 (2016).

22. Lipshutz, A. K. \& Gropper, M. A. Acquired neuromuscular weakness and early mobilization in the intensive care unit. Anesthesiology. 118 (1), 202-215 (2013).

23. Pohlman, M. C. et al. Feasibility of physical and occupational therapy beginning from initiation of mechanical ventilation. Crit Care Med. 38 (11), 2089-2094 (2010).

24. Bourdin, G. et al. The feasibility of early physical activity in intensive care unit patients: a prospective observational one-center study. Respir Care. 55 (4), 400-407 (2010).

25. Kortebein, P., Ferrando, A., Lombeida, J., Wolfe, R. \& Evans, W. J. Effect of 10 days of bed rest on skeletal muscle in healthy older adults. JAMA. 297 (16), 1772-1774 (2007).

26. Qun, L. Chen Rong Chang. Commentary on "Consensus Reporton Noninvasive Positive Pressure Ventilation". Chinese Journal of Practical Internal Medicine. 2009;(11):994-996

27. Hodgson, C. L. et al. Expert consensus and recommendations on safety criteria for active mobilization of mechanically ventilated critically ill adults. Crit Care. 18 (6), 658 (2014). 
28. Schweickert, W. D. et al. Early physical and occupational therapy in mechanically ventilated, critically ill patients: a randomised controlled trial. Lancet. 373 (9678), 1874-1882 (2009).

29. Wang Lijiao, Y., Xiaoqiong, D., Xi, W., Juan, S. \& Yaxu Effect observation of early pulmonary rehabilitation in patients with AECOPD complicating respiratory failure in respiratory care unit $J$ Mod Med Health, 2017; (24):3719-3722

30. Vestbo, J. et al. Global strategy for the diagnosis, management, and prevention of chronic obstructive pulmonary disease: GOLD executive summary. Am J Respir Crit Care Med. 187 (4), 347365 (2013).

31. Yosef-Brauner, O., Adi, N., Ben, S. T., Yehezkel, E. \& Carmeli, E. Effect of physical therapy on muscle strength, respiratory muscles and functional parameters in patients with intensive care unit-acquired weakness. Clin Respir J. 9 (1), 1-6 (2015).

32. Morris, P. E. et al. Early intensive care unit mobility therapy in the treatment of acute respiratory failure. Crit Care Med. 36 (8), 2238-2243 (2008).

33. Schaller, S. J. et al. Early, goal-directed mobilisation in the surgical intensive care unit: a randomised controlled trial. The Lancet. 388 (10052), 1377-1388 (2016).

34. Li, Y. \& Cuiping, C. Jiang Jinxia. Nursing research progress of mechanical ventilation patients' early mobility in ICU. Chin J Mod Nurs. 23 (20), 2692-2696 (2017).

35. Jiang Yulan, N. et al. Curative effect analysis of early rehabilitation activities for severe AECOPD patients during mechanical ventilation. Nurs. Res. 23 (20), 1994-1996 (2017).

36. Amidei, C. \& Sole, M. L. Physiological Responses to Passive Exercise in Adults Receiving Mechanical Ventilation. American Journal of Critical Care. 22 (4), 337-348 (2013).

37. De Jonghe, B. et al. Paresis acquired in the intensive care unit: a prospective multicenter study. JAMA. 288 (22), 2859-2867 (2002).

38. Hermans, G. \& Van den Berghe, G. Clinical review: intensive care unit acquired weakness. Crit Care. 19,274 (2015).

39. Mrozek, S. et al. Rapid onset of specific diaphragm weakness in a healthy murine model of ventilator-induced diaphragmatic dysfunction. Anesthesiology. 117 (3), 560-567 (2012).

40. Stevens, R. D. et al. Neuromuscular dysfunction acquired in critical illness: a systematic review. Intensive Care Med. 33 (11), 1876-1891 (2007).

41. Pu, L. et al. Weaning critically ill patients from mechanical ventilation: A prospective cohort study. $J$ Crit Care. 30 (4), 8627-8613 (2015).

42. Heunks, L. M. \& van der Hoeven, J. G. Clinical review: the ABC of weaning failure-a structured approach. Crit Care. 14 (6), 245 (2010).

43. Dreher, M., Kenn, K. \& Windisch, W. Non-invasive ventilation and physical exercise in patients with COPD. Pneumologie. 62 (3), 162-168 (2008).

44. Di Tays, M. K. et al. Lorenzo Valéria Amorim Pires, Noninvasive Ventilation as an Important Adjunct to an Exercise Training Program in Subjects With Moderate to Severe COPD. Respiratory Care, 2018, 
63(11):1388-1398.

45. Non-invasive ventilation. abolishes the IL-6 response to exercise in muscle-wasted COPD patients: a pilot study. Scand J Med Sci Sports. 24 (1), 136-143 (2014).

\section{Tables}

Table 1. The exercises (interventions) that the intervention group received.

\begin{tabular}{|c|c|}
\hline Step & Interventions \\
\hline \multicolumn{2}{|r|}{ Passive activities } \\
\hline 1 & Flexion and extension the patient's fingers ${ }^{32}$ \\
\hline 2 & Rotation of the wrist clockwise 32,35 \\
\hline 3 & Flexion and extension of the elbow 32,35 \\
\hline 4 & Abduction, flexion and rotation of each shoulder 32,35 \\
\hline 5 & Rotation of ankle joint clockwise ${ }^{32,35}$ \\
\hline 6 & Flexion and extension of knee joint ${ }^{32,35-36}$ \\
\hline 7 & Turning over in bed ${ }^{34-35}$ \\
\hline \multicolumn{2}{|r|}{ Initiative activities } \\
\hline 8 & Using soft elastic balls to train hand grip strength 34 \\
\hline 9 & Lifting the dumbbell ${ }^{34-35}$ \\
\hline 10 & Rotation of ankle joint (initiative) ${ }^{34}$ \\
\hline 11 & Flexion and extension of knee joint (initiative) ${ }^{34}$ \\
\hline 12 & Sit by the bed $28,33,35$ \\
\hline
\end{tabular}

Table 2. Baseline parameters and primary diagnosis of the participants. 


\begin{tabular}{|c|c|c|c|}
\hline & $\begin{array}{l}\text { Intervention } \\
\qquad(n=23)\end{array}$ & $\begin{array}{l}\text { Control } \\
(n=18)\end{array}$ & P value \\
\hline Gender (female number) & 17 (73.9\%) & $13(72.2 \%)$ & 1.00 \\
\hline Age (years) & $67.9 \pm 9.9$ & $67.6 \pm 10.2$ & 0.92 \\
\hline $\operatorname{BMI}\left(\mathrm{kg} / \mathrm{m}^{2}\right)$ & $20.7 \pm 1.9$ & $21.6 \pm 2.1$ & 0.17 \\
\hline APACHE II score & $18.0 \pm 4.6$ & $16.3 \pm 4.5$ & 0.76 \\
\hline Creatinine (mmol/L) & $58(47-71)^{a}$ & $56.5(43-78)^{a}$ & 0.85 \\
\hline Leukocyte $\left(10^{9} / \mathrm{L}\right)$ & $7.9(7.1-9.5)^{a}$ & $9.3(6.2-10.8)^{a}$ & 0.39 \\
\hline \multicolumn{4}{|c|}{ Primary diagnosis on admission } \\
\hline Acute exacerbation of COPD & $18(78.3 \%)$ & $16(88.9 \%)$ & 0.63 \\
\hline Acute lung injury & $5(21.7 \%)$ & $2(11.1 \%)$ & 0.61 \\
\hline \multicolumn{4}{|l|}{ Risk factors } \\
\hline Cardiac disease & $11(47.8 \%)$ & $12(66.7 \%)$ & 0.23 \\
\hline Hypertension & $7(38.9 \%)$ & $7(30.4 \%)$ & 0.57 \\
\hline Diabetes Mellitus & $8(44.4 \%)$ & $6(26 \%)$ & 0.22 \\
\hline Bronchial asthma & $1(5.6 \%)$ & $3(13.0 \%)$ & 0.19 \\
\hline
\end{tabular}

Note: ${ }^{a}$ Kolmogorov-Smirnov test, p <0.05. "BMI" = "Body Mass Index", "APACHE” = "Acute Physiology and Chronic Health Evaluation", "COPD” = "chronic obstructive pulmonary diseases”

Table 3. The outcome measures.

\begin{tabular}{|c|c|c|c|}
\hline & $\begin{array}{l}\text { Intervention } \\
\qquad(n=23)\end{array}$ & $\begin{array}{l}\text { Control } \\
(n=18)\end{array}$ & $P$ value \\
\hline Incidence of ICU-acquired weakness & $4(17.4 \%)$ & $9(50 \%)$ & $0.026 *$ \\
\hline The length of ICU stay (days) & $6.0(4.3-7.1)^{\mathrm{a}}$ & $7.8(5.0-15.7)^{a}$ & $0.038 *$ \\
\hline Duration of ventilation (days) & $2.1(1.3-4.7)^{\mathrm{a}}$ & $4.0(2.0-6.4)^{a}$ & $0.019 *$ \\
\hline ICU mortality & $0(0 \%)$ & $1(5.6 \%)$ & 0.439 \\
\hline Increase of right hand-grip force (LB) & $4.9(2.2-12.1)^{a}$ & $-2.2(-7.7-2.0)^{a}$ & $0.00 *$ \\
\hline Increase of left hand-grip force (LB) & $3.1(0-6.8)^{\mathrm{a}}$ & $-5.6(-5.7-3.7)^{a}$ & $0.047 *$ \\
\hline
\end{tabular}


Note: ${ }^{a}$ Kolmogorov-Smirnov test, $\mathrm{p}<0.05 ; * \mathrm{p}<0.05$.

\section{Figures}

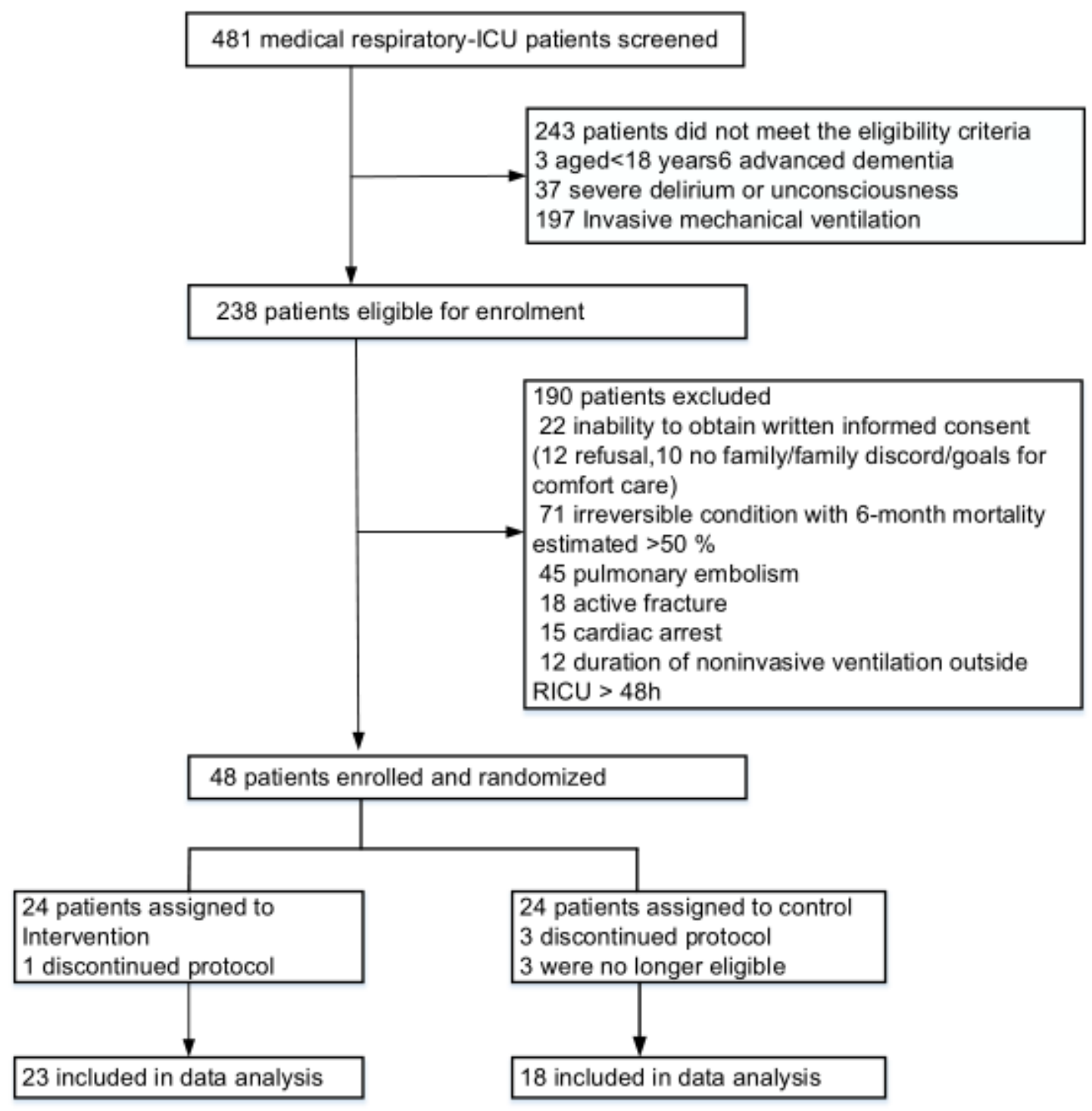

\section{Figure 1}

Trial profile. $(\mathrm{RICU}=$ Respiratory Intensive Care Unit) 